\title{
Metamaterials-based sensor to detect and locate nonlinear elastic sources
}

\author{
Antonio S. Gliozzi, ${ }^{1}$ Marco Miniaci, ${ }^{2}$ Federico Bosia, ${ }^{2}$ Nicola M. Pugno, $, 3,4$ \\ and Marco Scalerandi ${ }^{1}$ \\ ${ }^{1}$ Department of Applied Science and Technology, Politecnico di Torino, Corso Duca degli Abruzzi 24, \\ 10129 Torino, Italy \\ ${ }^{2}$ Department of Physics, University of Torino, Via Pietro Giuria 1, 10125 Torino, Italy \\ ${ }^{3}$ Laboratory of Bio-Inspired and Graphene Nanomechanics, Department of Civil, Environmental and \\ Mechanical Engineering, University of Trento, Via Mesiano 77, 38123 Trento, Italy \\ ${ }^{4}$ Center for Materials and Microsystems, Fondazione Bruno Kessler, Via Sommarive 18, 38123 Povo (Trento), \\ Italy \\ ${ }^{5}$ School of Engineering and Materials Science, Queen Mary University of London, Mile End Road, \\ London E1 4NS, United Kingdom
}

(Received 18 May 2015; accepted 11 October 2015; published online 21 October 2015)

\begin{abstract}
In recent years, acoustic metamaterials have attracted increasing scientific interest for very diverse technological applications ranging from sound abatement to ultrasonic imaging, mainly due to their ability to act as band-stop filters. At the same time, the concept of chaotic cavities has been recently proposed as an efficient tool to enhance the quality of nonlinear signal analysis, particularly in the ultrasonic/acoustic case. The goal of the present paper is to merge the two concepts in order to propose a metamaterial-based device that can be used as a natural and selective linear filter for the detection of signals resulting from the propagation of elastic waves in nonlinear materials, e.g., in the presence of damage, and as a detector for the damage itself in time reversal experiments. Numerical simulations demonstrate the feasibility of the approach and the potential of the device in providing improved signal-to-noise ratios and enhanced focusing on the defect locations. (C) 2015 AIP Publishing LLC. [http://dx.doi.org/10.1063/1.4934493]
\end{abstract}

Acoustic metamaterials (AMMs) are artificially structured composite materials that enable manipulation of the dispersive properties of vibrational waves. Generally, they are periodic distributions of cavities or inclusions (scatterers) embedded in a matrix. ${ }^{1}$ Among their unique vibrational characteristics, the attractive property of such structures to act as stop-band filters can be exploited to attenuate mechanical waves over entire frequency bands, commonly known as band gaps (BGs). As a consequence, appropriate configurations of the metamaterial structure allow focusing of energy in selected frequency ranges. ${ }^{2}$

These two main properties make the AMMs potentially interesting for applications in nonlinear acoustics and structural health monitoring. ${ }^{3,4}$ A mechanical wave that travels through a damaged structure generally interacts with interfaces, crack tips, delaminations, etc., acquiring and conveying information about the specimen integrity or type of damage. Nonlinear phenomena emerging from these interactions are by far the most interesting features, both from the theoretical and the applicative point of view. ${ }^{5-8}$ In the presence of large stress or strain values, the linear approximation in the stressstrain relation (Hooke's law) is no longer valid due to the anomalous local dynamics of material portions (typically defects). ${ }^{9}$ This gives rise to a spectral modification in the output response, generating multiple harmonics of the input solicitation frequency, nonlinear attenuation effects, and changes in the wave velocity or in the resonance frequency. The nonlinear techniques that have been developed for damage detection are in general designated as Nonlinear Elastic Wave Spectroscopy (NEWS). ${ }^{10-12}$

One important application of the NEWS techniques is damage locating through nonlinear Time Reversal (TR) experiments. ${ }^{13-15}$ The TR procedure consists of re-injecting a time-reversed signal into the sample: due to the invariance of the equation of motion under a time inversion $(t \rightarrow-t)$, the re-injected wave back-propagates through the medium retracing the incoming multiple scattering paths and ultimately refocuses at the original source location. The basic idea of nonlinear TR is that the defects, by scattering incident elastic waves, act as active sources of higher order harmonics. It is then possible to filter the recorded signal and time-reverse only the part of the signal generated by the defect.

Despite the considerable interest attracted by the NEWS and nonlinear TR techniques and the resulting advances in recent years, ${ }^{16,17}$ several aspects remain to be addressed. Since the information is carried by higher order harmonics and their spectral weight is very low compared to that of the fundamental (at most of the order of a few percent), an increase of the signal to noise ratio is of primary importance $^{18-20}$ and it is necessary to amplify signals due to the nonlinear effects that are often submerged below the noise level. $^{21}$

In this work, we propose to integrate standard piezoelectric sensors with the AMM-based structures, exploiting their capability of naturally filtering out or reflecting incident mechanical waves in the prescribed frequency ranges, thus enhancing the efficiency in sensing higher harmonics and focusing on the nonlinear TR. The advantages with respect to traditional digital (post-processing) filtering performed with standard piezoelectric sensors are discussed and an application to NEWS-TR is presented.

An example of such a meta-device is shown schematically in Fig. 1(a). The device includes a chaotic cavity, ${ }^{22,23}$ 


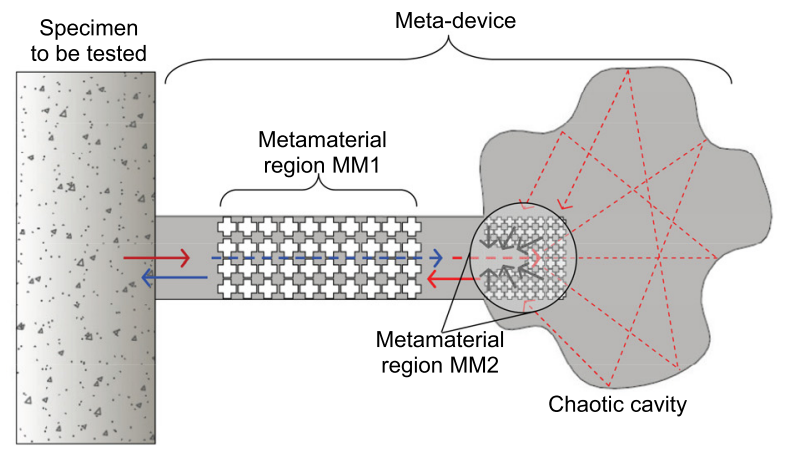

(a)

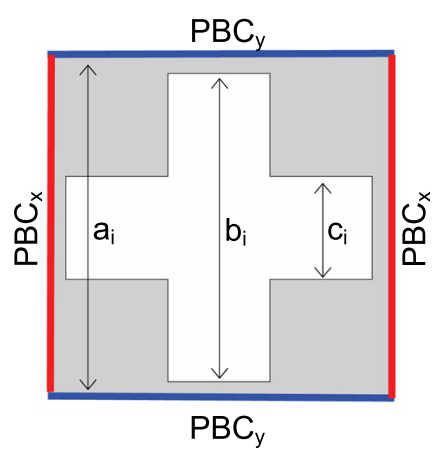

(b)

FIG. 1. (a) Schematic representation of the meta-device composed of a filtering metamaterial region (MM1 region) and a chaotic cavity with an additional focusing metamaterial-based structure (MM2 region). (b) Cross-section of the unit cells for the MM1 and MM2 regions ( $i=1$ and 2$)$ along with their periodic boundary conditions in the x-direction $\left(P B C_{x}\right)$ and y-direction $\left(P B C_{y}\right)$, respectively. Parameters are $a_{1}=1.8 \mathrm{~mm}, b_{1}=0.9 \cdot a_{1}, c_{1}=0.4 \cdot a_{1}$, and $a_{2}=1 \mathrm{~mm}$, $b_{2}=0.9 \cdot a_{2}, c_{2}=0.3 \cdot a_{2}$.

which has been introduced in NEWS-TR applications in the past to enhance, through its irregular geometry, multiple scattering and reflections. A piezoelectric transducer placed on the surface of the cavity acts as a receiver (and as an actuator in the backward propagation in a TR experiment). Two metamaterial regions, indicated as MM1 and MM2 in Fig. 1(a), are integrated in the meta-device: the first to filter out the fundamental frequency of the incident wave, which does not contain the nonlinear signature of damage, and the second to selectively focus a higher harmonic component on the receiver, thus enhancing the amplitude of the nonlinear components of the recorded signal with respect to the noise level and to the other frequency components. The two regions (MM1 and MM2) have different roles of filtering (barrier) or focusing (mirror) elastic waves, depending on their geometrical configuration and location. Both exploit the property of AMMs to selectively reflect a certain range of frequencies, depending on the lattice parameters. In particular, in our configuration, MM2 has been designed to reflect the second order harmonics and to focus them in the geometrical centre of the mirror, where the receiver is placed.

In order to demonstrate the enhancement of the sensitivity provided by the AMMs, the meta-device performance is analysed using 2D simulations on a section of the coupled system (consisting of the sample and the device). The metadevice is modelled as an isotropic aluminium plate (density $\rho=2700 \mathrm{~kg} / \mathrm{m}^{3}$, Lamé elastic constants $\lambda=51.08 \mathrm{GPa}$, $\mu=26.31 \mathrm{GPa}$ ) with periodic arrangements of cross-shaped cavities. This configuration is chosen due to the good compromise obtained between small solid/void ratio and large BGs. ${ }^{24}$ The so-called unit cells, i.e., the smallest geometries that allow the full computation of the AMM dispersion diagrams, are illustrated in Fig. 1(b), for the MM1 and MM2 regions ( $i=1$ and 2, respectively). The band structure is derived assuming periodic boundary conditions $(P B C x$ and $P B C y)$ at the edges of the cells. The resulting eigenvalue problem is solved along the three symmetry paths of the first irreducible Brillouin zone $\Gamma-X-M$ using finite element (FE) simulations and exploiting the Bloch-Floquet theorem $^{25-27}$ with the aid of the commercial software Comsol MultiPhysics. ${ }^{28}$ The unit cell domains are modelled under the 2D plane strain assumption and meshed by means of 3-node triangular elements of maximum size $L_{F E}=0.05 \mathrm{~mm}$ to provide accurate eigensolutions up to the maximum frequency of $3.5 \mathrm{MHz}$. Fig. 2 presents the band structures for the filtering metamaterial (MM1) and the focusing metamaterial (MM2) in terms of reduced wavevector $k^{*}=\left[k_{x} \cdot a / \pi\right.$; $\left.k_{y} \cdot a / \pi\right]$, where $k_{x}$ and $k_{y}$ are the wavevectors in the $x$ and $y$ (a)

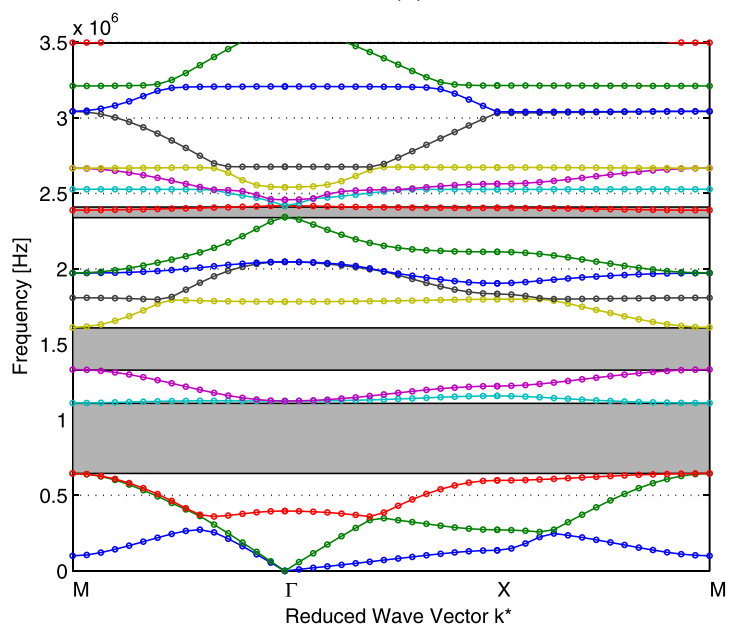

(b)

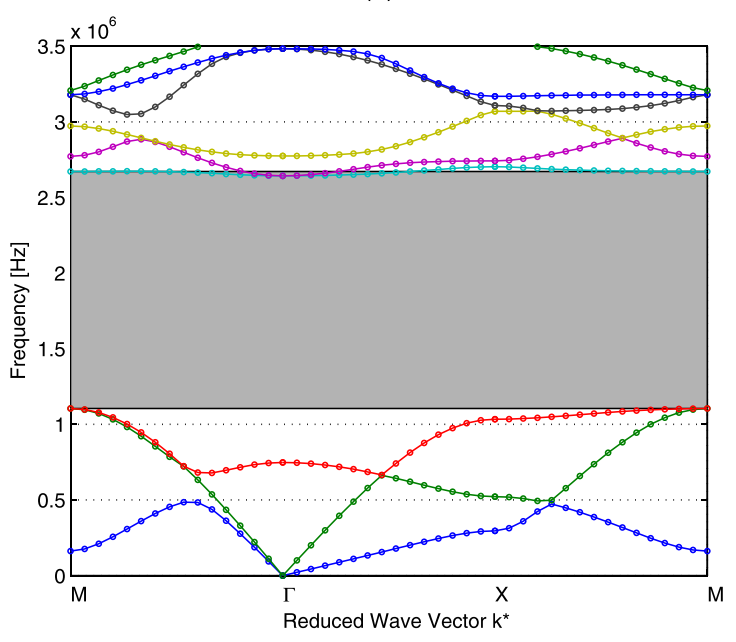

FIG. 2. Dispersion diagrams for unit cells described in Fig. 1(b) for (a) $i=1$ and (b) $i=2$. 
directions, respectively. For the MM1 structure, three complete BGs (light grey rectangles) exist in the $0-3.5 \mathrm{MHz}$ frequency range, with a larger complete $\mathrm{BG}$ extending from $0.64 \mathrm{MHz}$ to $1.12 \mathrm{MHz}$ (Ref. 29) (Fig. 2(a)). A similar dispersion diagram is obtained for the MM2 structure, with a single $\mathrm{BG}$ centred around $f=2 \mathrm{MHz}$ (Fig. 2(b)).

The considered sample containing a nonlinear defect consists of a 2D isotropic plate of dimensions $14 \times 3 \mathrm{~cm}^{2}$, with the following Lamé constants: $\lambda=5.00 \mathrm{GPa}$ and $\mu=8.90 \mathrm{GPa}$. The density is $\rho=2400 \mathrm{~kg} / \mathrm{m}^{3}$. The meta-device is coupled to the sample (under the hypothesis of ideal contact) with a contact length of $d=1 \mathrm{~cm}$. A small $\left(1 \times 1 \mathrm{~mm}^{2}\right)$ hysteretic nonlinear square defect is located at $P=(4,2) \mathrm{cm}$. A nonclassical nonlinear model developed by some of the authors, ${ }^{12,30,31}$ implemented using a Preisach-Mayergoyz ${ }^{32}$ space representation, based on a multi-state elastic model, is adopted to simulate the nonlinear elastic response of the defect (the model is briefly described in the supplementary material ${ }^{29}$ ).

We first analyze the filtering properties of the metadevice described in Fig. 1(a), comparing it to the performance of a chaotic cavity without metamaterial regions MM1 and MM2. A Gaussian modulated plane wave with a central frequency falling in the first $\mathrm{BG}$ of the filtering metamaterial $\left(f_{c}=1 \mathrm{MHz}\right)$ is injected from a transducer placed on the left edge of the sample (i.e., on the opposite side with respect to the meta-device) and the propagation in the specimen is simulated. The interaction of the propagating wave with the

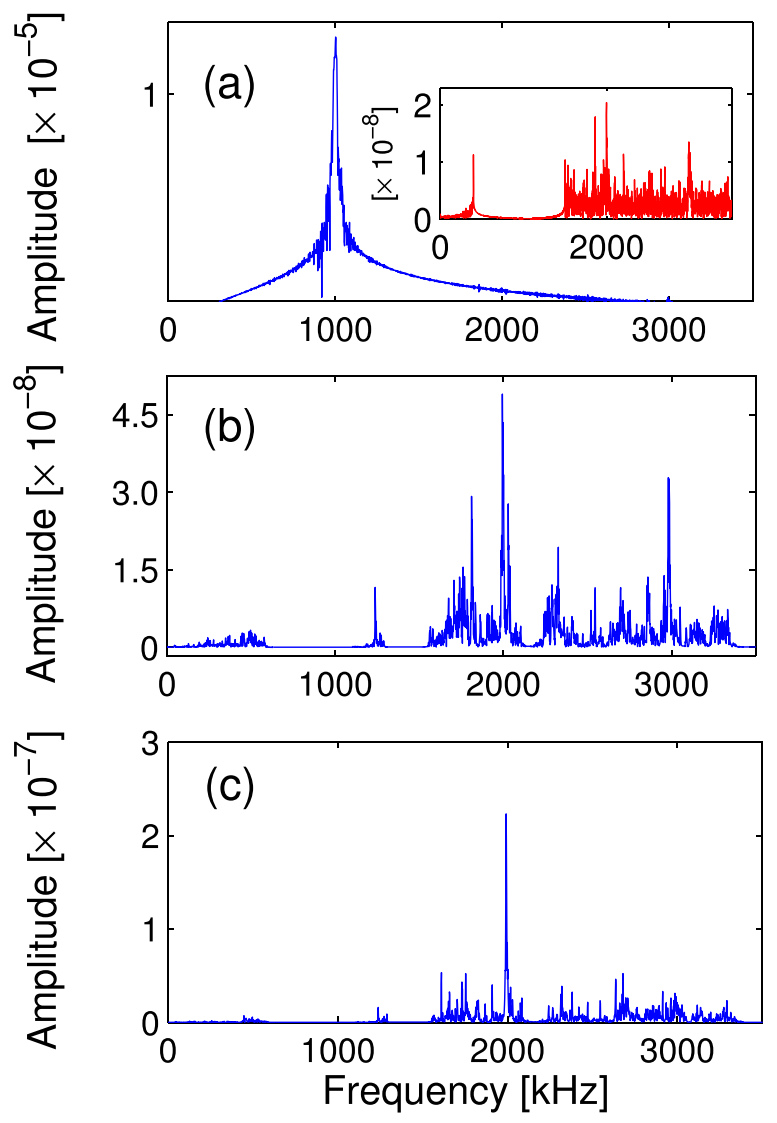

FIG. 3. FFT magnitude of the detected signal in the meta-device shown in Fig. 1(a): (a) replacing MM1 and MM2 regions with a homogeneous material (inset shows results of digital filtering around $1 \mathrm{MHz}$ ); (b) with only the MM1 region, replacing MM2 with a homogeneous material; and (c) with both MM1 and MM2 regions. hysteretic defect generates higher order harmonics. Across the contact surface between sample and device, the wave propagates and interacts with the geometrical constraints (boundaries) of the chaotic cavity, without any change in the frequency spectrum. A long time signal is detected at the receiver position. ${ }^{29}$

In Fig. 3, the spectrum of the velocity signals obtained in different cases is shown and compared. As expected, when only a chaotic cavity transducer is used (i.e., replacing the metamaterial regions MM1 and MM2, in Fig. 1(a) with homogeneous aluminum regions), the spectral component at the frequency of the source dominates (Fig. 3(a)). If a digital filter centred around the fundamental frequency is applied (see inset), the second and third harmonics become visible. Addition of the MM1 region to the cavity leads to a similar spectrum, as shown in Fig. 3(b), with the spectral components around the second and third harmonics that are slightly more evident than in the previous case. Thus, the sensor equipped with a single metamaterial region (MM1) can act as an efficient analog filter. Furthermore, addition of the second metamaterial region (MM2) leads to both filtering and focusing, since only the second harmonic is detected, as shown in Fig. 3(c). Of course, the second metamaterial region could be designed to act as a focusing mirror for any chosen higher order harmonic. The detected amplitude is much larger (about 5 times larger than in previous cases), showing the advantages of an increased signal to noise ratio and of selective (almost monochromatic) filtering, even if the focusing mirror is not designed with an optimal shape. Notice that the design of spherical-shape metamaterial mirrors is not trivial and might lead to further improvements for the detection of the nonlinear signature in the detected time signals (work is in progress on this topic). The improvements obtained using the proposed device with respect to conventional digital filtering techniques, both in amplitude and quality of the Fast Fourier Transform (FFT), become particularly important when time signals are affected by intrinsic noise and/or when direct analysis of the time signals is required for a fast and simple online inspection. In this case, time signals with amplitudes above the noise level trigger an on/off threshold for the recognition of the presence of damage in the sample (see discussion in the supplementary material ${ }^{29}$ ).

Finally, an application of the meta-device described in Fig. 1(a) is presented to demonstrate the possibility of employing it for NEWS-TR. The filtering process in NEWSTR is normally performed in a post-processing procedure. Instead, using the meta-device we obtain natural filtering by the AMM that is transparent only to the super-harmonics generated by the defect. The signal recorded by the sensor in the chaotic cavity can thus be readily inverted and retransmitted into the sample.

As in the case of traditional TR imaging procedures, the experiment is performed in two steps: first, in forward propagation, the sample is illuminated by a perturbation (8-cycles of a Gaussian modulated wave), with central frequency of $f_{c}=1 \mathrm{MHz}$ and a time length of $\tau=0.01 \mathrm{~ms}$. Then, in backward propagation, the recorded signal of total length $\Delta t=3 \mathrm{~ms}$ is time inverted and re-injected from the location of the sensor that can also be used as an actuator. 


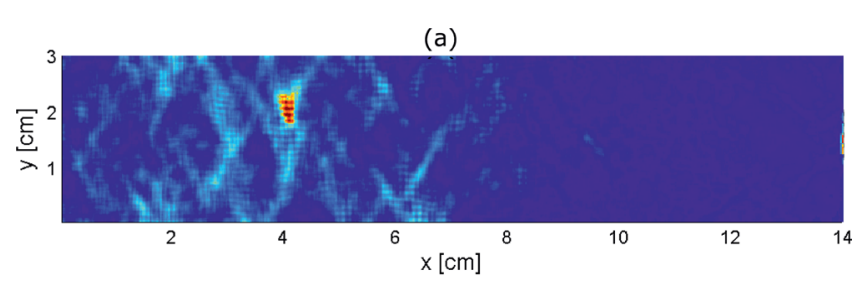

(b)

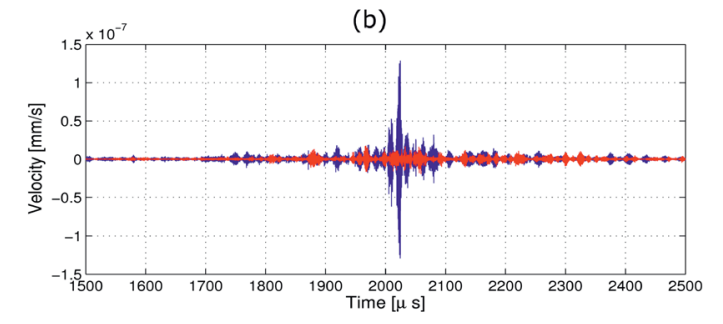

FIG. 4. Results of the Time Reversal experiment. (a) Map of the wavefield at the focal time. (b) The TR temporal signals are recorded in two different points of the sample: one (blue line) in the position of the nonlinear defect (the focusing of the signal is evident), the other (red line) in a generic point of the sample (no time focusing is observed).

The backward propagation, spatial focusing of the wave field and temporal compression of the signal, is expected at the defect location. The velocity of each node of the grid is recorded at each time step of the simulation. This gives a map of the wave field, similar to that obtained in the experimental results using a scanning laser Doppler vibrometer. At the focal time, the spatial map of the recorded velocities reveals focusing at the position of the defect, with a considerable concentration of energy, as shown in Fig. 4(a). Focusing is also obtained in the time domain, as can be seen by plotting the recorded time signal. In Fig. 4(b), the time evolution of the velocities calculated at two different nodes is reported: the first is located at the focal point, at the centre of the nonlinear scatterer (blue line), and shows excellent time compression (focusing); the second is far from the focal point (red line) and displays an incoherent and defocused signal. Notice that multiple scattering due to backward propagation of the wavefield through the AMM increases the efficiency of TR and provides a high degree of focusing. To confirm the efficiency of the proposed approach, we also performed simulations on the same structure without the AMM, using other filtering methods (e.g., Phase Inversion ${ }^{33}$ ) obtaining very similar results. ${ }^{29}$

In conclusion, we have proposed to combine in a single device the bandstop filter properties of a metamaterial with the high sensitivity of a chaotic cavity for the detection of harmonics generated by defect-related nonlinearities. We have demonstrated in numerical simulations that the proposed device allows selective filtering of single harmonic components, without the need of digital post-processing and with increased nonlinear signal component amplitudes at the receiver position. The simplicity in design and potential low production costs could make the proposed sensor a valid alternative to standard piezoelectric transducers for nonlinear acoustics experiments. In addition, other acoustic metamaterial properties such as tunability, cloaking, or wave guiding could potentially be exploited to further enhance the sensor capabilities or even multifunctionality.
N.M.P. was supported by the ERC (StG Ideas 2011 No. 279985 BIHSNAM, PoC 2013-1 No. 619448 REPLICA2, PoC 2013-2 No. 632277 KNOTOUGH), by the EC Graphene Flagship (WP10, No. 604391), and by the Provincia Autonoma di Trento ("Graphene nanocomposites," No. S116/ 2012-242637 and reg. delib. No. 2266). M.M. and F.B. were supported by BIHSNAM.

${ }^{1}$ P. A. Deymier, Acoustic Metamaterials and Phononic Crystals, Springer Series in Solid-State Sciences (Springer, Berlin, Heidelberg, 2013).

${ }^{2}$ Y. Pennec, J. O. Vasseur, B. Djafari-Rouhani, L. Dobrzyński, and P. A. Deymier, Surf. Sci. Rep. 65(8), 229-291 (2010).

${ }^{3}$ J. Blitz and G. Simpson, Ultrasonic Methods of Non-Destructive Testing (Springer-Verlag, New York, 1991).

${ }^{4}$ P. S. Ma, Y. E. Kwon, and Y. Y. Kim, Appl. Phys. Lett. 103, 151901 (2013).

${ }^{5}$ O. Buck, W. L. Morris, and J. M. Richardson, Appl. Phys. Lett. 33, 371 (1978).

${ }^{6} \mathrm{~K}$. Naugolnykh and L. Ostrovsky, Nonlinear Wave Processes in Acoustic (Cambridge University Press, 1998).

${ }^{7}$ C. Pecorari, J. Acoust. Soc. Am. 113(6), 3065-3072 (2003).

${ }^{8}$ M. Scalerandi, A. S. Gliozzi, C. L. E. Bruno, and P. Antonaci, Phys. Rev. B 81, 104114 (2010).

${ }^{9}$ I. Solodov, D. Döring, and G. Busse, J. Mech. Eng. 57(3), 169-182 (2011).

${ }^{10}$ C. L. E. Bruno, A. S. Gliozzi, M. Scalerandi, and P. Antonaci, Phys. Rev. B 79, 064108 (2009).

${ }^{11}$ R. A. Guyer and P. A. Johnson, Nonlinear Mesoscopic Elasticity: The Complex Behaviour of Rocks, Soil, Concrete (Wiley, 2009).

${ }^{12}$ P. P. Delsanto and M. Scalerandi, J. Acoust. Soc. Am. 104, 2584-2591 (1998).

${ }^{13}$ T. Goursolle, S. Callé, S. Dos Santos, and O. Bou Matar, J. Acoust. Soc. Am. 122, 3220-3229 (2007).

${ }^{14}$ A. S. Gliozzi, M. Griffa, and M. Scalerandi, J. Acoust. Soc. Am. 120(5), 2506-2517 (2006).

${ }^{15}$ T. J. Ulrich, P. A. Johnson, and R. A. Guyer, Phys. Rev. Lett. 98, 104301 (2007).

${ }^{16}$ T. J. Ulrich, A. M. Sutin, T. Claytor, P. Papin, P.-Y. Le Bas, and J. A. TenCate, Appl. Phys. Lett. 93, 151914 (2008).

${ }^{17}$ M. Scalerandi, A. S. Gliozzi, C. L. E. Bruno, and K. Van Den Abeele, J. Phys. D: Appl. Phys. 41, 215404 (2008).

${ }^{18}$ G. D. Connolly, J. Li, and S. I. Rokhlin, NDT\&E Int. 55, 47-56 (2013).

${ }^{19}$ M. Bentahar, R. El Guerjouma, S. Idijmarene, and M. Scalerandi, J. Appl. Phys. 113, 043516 (2013).

${ }^{20}$ M. Scalerandi, A. S. Gliozzi, C. L. E. Bruno, D. Masera, and P. Bocca, Appl. Phys. Lett. 92, 101912 (2008).

${ }^{21}$ I. Solodov, J. Bai, S. Bekgulyan, and G. Busse, Appl. Phys. Lett. 99, 211911 (2011).

${ }^{22}$ C. Draeger and M. Fink, Phys. Rev. Lett. 79, 407 (1997).

${ }^{23}$ O. Bou Matar, Y. Li, V. L. Preobrazhensky, and P. Pernod, J. Acoust. Soc. Am. 123, 3830 (2008).

${ }^{24}$ Y.-F. Wang, Y.-S. Wang, and X.-X. Su, J. Appl. Phys. 110, 113520 (2011).

${ }^{25}$ L. Brillouin, Wave Propagation in Periodic Structures: Electric Filters and Crystal Lattices, Chemische Reihe. Lehrbucher und Monographien aus dem Gebiete der exakten Wissenschaften (Dover Publications, 1953).

${ }^{26}$ M. Collet, M. Ouisse, M. Ruzzene, and M. N. Ichchou, Int. J. Solids Struct. 48, 2837-2848 (2011).

${ }^{27}$ M. Miniaci, A. Marzani, N. Testoni, and L. De Marchi, Ultrasonics 56, 251-259 (2015).

${ }^{28}$ See www.comsol.com for Comsol multiphysics—release 4.3a, 2012.

${ }^{29}$ See supplementary material at http://dx.doi.org/10.1063/1.4934493 for the description of this aspect.

${ }^{30}$ A. S. Gliozzi and M. Scalerandi, J. Acoust. Soc. Am. 136(4), 1530 (2014).

${ }^{31}$ P. P. Delsanto and M. Scalerandi, Phys. Rev. B 68, 064107 (2003).

${ }^{32}$ I. D. Mayergoyz, J. Appl. Phys. 57, 3803 (1985).

${ }^{33}$ P.-Y. Le Bas, K. Van den Abeele, S. Dos Santos, T. Goursolle, and O. Bou Matar, "Experimental analysis for nonlinear time reversal imaging of damaged materials," in Proceedings of the 9th European Conference on NonDestructive Testing, Berlin (ECNDT, 2006). 